Rev. salud pública. 10 sup (1): 133-145, 2008

\title{
El Seguro Popular de Salud en México: Pieza Clave de la Inequidad en Salud
}

\author{
Popular Health Insurance: key piece of inequity in health in Mexico
}

\author{
Silvia Tamez González y Catalina Eibenschutz
}

Departamento de Atención a la Salud. Área de investigación Estado y servicios de salud. Universidad Autónoma Metropolitana. México, D.F. stamez@correo.xoc.uam.mx, ceibenschutz@yahoo.com.mx

Recibido 8 Enero 2007/Enviado para Modificación 2 Mayo 2008/Aceptado 29 Agosto 2008

\section{RESUMEN}

El objetivo central de este trabajo es presentar un análisis de la situación actual del Sistema Nacional de Salud mexicano, resultado de las sucesivas reformas realizadas desde la década de los ochenta. Interesa de manera especial, enfatizar el papel que ha jugado el Seguro Popular de Salud (SPS) como pieza clave de la mercantilización de la atención médica. Para tal efecto, en la primera parte se presentan los principales antecedentes de los cambios realizados durante las dos últimas décadas del siglo pasado, y en la segunda se analiza la situación actual a partir del inicio del nuevo milenio. El análisis se centra en una evaluación inicial de los alcances y limitaciones del Seguro Popular de Salud bajo la perspectiva de la equidad del acceso a la atención médica. El análisis concluye que debido a que no hay una perspectiva médica en las reformas estructurales, este seguro representa un programa discrecional, presidencial y focalizado que resta fondos a las instituciones grandes de la seguridad social, obligándolas, en muchos casos, a realizar adecuaciones presupuestarias en detrimento de la calidad de la atención. Esta situación constituye, en el futuro inmediato, una segmentación del Sistema Nacional de Salud que determinará nuevas condiciones diferenciales del acceso de la población a los servicios médicos, aumentará la inequidad en salud y contribuirá a incrementar la gran desigualdad social prevaleciente en México.

Palabras Clave: Equidad, acceso, atención médica, reforma sanitaria, seguridad social (fuente: DeCS, BIREME).

\section{ABSTRACT}

This work is aimed at presenting an analysis of the Mexican health system's current situation resulting from successive reforms which have been carried out since the 1980s. Special interest is placed on the role which the Seguro Popular de Salud (SPS - a "popular", meaning universal, health insurance plan) has played, being a key piece in commercialising medical attention. The first part of this work thus presents the main antecedents for the changes made during the last two decades of the last century and analyses the current situation since the start of the new millennium. 
Such analysis is centred on an initial evaluation of the Seguro Popular de Salud's scope and limitations from the perspective of equity in gaining access to medical attention. The analysis concludes that due to a medical perspective not having been present in the structural reforms, then this insurance policy represents a discretional, presidential and focalised programme taking funds away from the large social security institutions, obligating them (in many cases) to make budgetary adaptations to the detriment of providing quality attention. This situation will constitute (in the immediate future) a segmentation of the National Health System which will determine new conditions regarding the population's differential access to medical services, increase inequity in health and contribute towards increasing the great social inequality prevailing in México.

Key Words: Equity, access, medical attention, sanitary reform, social security (source: MeSH. NLM).

La reforma de los servicios de salud en México. Principales antecedentes

$\mathrm{E}$ n México, desde inicios de la década de los ochenta se empezaron a perfilar las intenciones gubernamentales de transformar los servicios de salud bajo la propuesta del establecimiento de una coordinación entre las instituciones del Sistema Nacional de Salud. Sin embargo, esta iniciativa no prosperó debido a que el Instituto Mexicano del Seguro Social (en ese entonces la institución más fuerte del sector), no colaboró con el ministerio de salud en esta iniciativa.

Durante el mandato de Carlos Salinas de Gortari (1988-1994), se pusieron las bases para iniciar el inexorable proceso de desmantelamiento de la seguridad social, pues se eliminó el papel del Instituto Mexicano del Seguro Social (IMSS) como responsable de la administración de fondos y pagador de las pensiones de los trabajadores del sector industrial, al asignar estas tareas a las Administradoras de Fondos para el Retiro (AFORES) y a las aseguradoras. Con estas medidas, se inaugura la administración y el control privado sobre los fondos de pensiones y se establecen las bases para la transferencia masiva de fondos del sector público al privado, el fortalecimiento de los grupos financieros y la mercantilización del sistema de pensiones (1). En este sexenio, también se perfilaron con mayor fuerza las acciones de focalización contra la pobreza, en las que se incluyeron diversas actividades en salud que dependían directamente del Ejecutivo Federal. Sin embargo, es hasta mediados de la década de los noventa, durante el sexenio de Ernesto Zedillo, que se expresan con mayor claridad las intenciones del Estado mexicano de adoptar al pie de la letra las recomendaciones del Banco Mundial y del Banco Interamericano de Desarrollo. 
Estas recomendaciones se orientaron a realizar la primera fase de reforma de los servicios de salud, para lo cual, era necesario fracturar las bases de la seguridad social, que ya por entonces atendía alrededor del 50\% de la población mexicana. Así, durante la década de los noventa, el gobierno continúa la reforma del sistema de salud mexicano, favoreciendo el reforzamiento y la creación de las condiciones necesarias para la mercantilización de la atención médica. Las dos acciones fundamentales para lograrlo fueron el debilitamiento de la seguridad social mediante las modificaciones a la Ley del IMSS (2) y la reorganización de los servicios que atienden a la población no asegurada (3). Los cambios en este periodo fueron muchos y muy diversos, se puede decir que sentaron las bases para la expresión plena de los verdaderos objetivos de la reforma de los servicios de salud: la emergencia de la anhelada mezcla pública-privada, como forma de garantizar el flujo de recursos del sector público de la atención médica al sector privado.

En la presente década, la identidad del Programa Nacional de Salud 20012006 con el plan del gobierno anterior, así como las acciones realizadas por el gobierno actual, expresan claramente que la reforma de los servicios de salud en México forma parte de la reforma del Estado mexicano. Desde luego, es necesario tener en cuenta que una aplicación tan radical de esta reforma fue posible gracias a la ausencia de participación de la mayoría de los actores sociales involucrados.

El nuevo siglo: continuidad de las tendencias de reforma

A partir del año 2000, durante el gobierno de Vicente Fox, se diseña y aplica el Programa Nacional de Salud 2001-2006, que fue titulado "La democratización de la salud en México", el cual profundiza las estrategias reseñadas anteriormente. En él se plantea la necesidad de "alinear las políticas fiscales con las políticas de salud mediante el estímulo al prepago por la atención a la salud” (4).

En el año 2001, el Banco Mundial propone medidas para prácticamente todos los ámbitos sociales y económicos del país, a partir de una agenda de desarrollo de cinco puntos. En ese documento se plantea un ordenamiento general que sin duda atañe a la reorganización de los servicios de salud, pues en él se plantea la necesidad de: "Redoblar los esfuerzos para acelerar el crecimiento y la competitividad en el largo plazo, estableciendo un sistema financiero sólido que estimule la inversión privada y proteja los ahorros de los mexicanos. Se recomienda asimismo eliminar las barreras a los flujos de capital 
privado liberalizando en forma gradual las áreas que actualmente se encuentran dominadas por el sector público o son monopolios cuasi-públicos”(5).

En el documento "La Reforma de la Salud en México”(6), basado en el Informe sobre la Salud en el Mundo de 2000 (7), se presentan 4 acciones prioritarias para lograr las metas del PNS 2001-2006, a saber: a) establecer el seguro nacional de salud (actualmente Seguro Popular de Salud-SPS); b) separar el financiamiento de la prestación de servicios; c) incentivar la participación del sector privado en el cuidado de la salud y d) crear un fondo nacional de salud pública (8).

La separación de funciones se centró en el cambio del papel del ministerio de salud, limitándolo a la recaudación y normatividad de las demás instancias del SNS, al margen de la prestación de servicios. En la seguridad social, las cuotas del seguro de enfermedad y maternidad fueron transferidas a un organismo autónomo, denominado Fondo Solidario de Salud (FSS), orientado a asegurar el "techo financiero" y transferir los recursos a las administradoras de salud, las cuales pueden ser públicas o privadas.

La principal razón que fue esgrimida a favor de incentivar la participación privada en la prestación de servicios de salud fue la saturación de los servicios del IMSS. Por su parte, la propuesta gubernamental, con claros tintes privatizadores, señala que ante la imposibilidad de atender a la "nueva población derechohabiente”, constituida por los grupos cotizantes más altos y por los trabajadores de la economía informal, se debe utilizar la infraestructura disponible a través de establecimientos privados mediante un financiamiento público. En la actualidad este proceso de transformaciones tiene como principal pivote la expansión del SPS pues, como ya se dijo anteriormente, este programa garantiza, masivamente, la expansión de la mezcla público-privada de la atención médica.

Actualmente, el Sistema Nacional de Salud está constituido por tres subsistemas. El primero representado por la seguridad social laboral (Instituto Mexicano del Seguro Social, Instituto de Seguridad y Servicios Sociales de los Trabajadores del Estado-ISSSTE, Petróleos Mexicanos, etc.); el segundo, correspondiente a servicios para población no asegurada por la seguridad social (Secretaría de Salud y Servicios Estatales de Salud); y el privado, conformado por compañías aseguradoras, empresas médicas y prestadores pequeños y privados. Estos subsistemas fueron constituidos desde formas de financiamiento específicas. El primero, por el Instituto de Seguridad Social, financiado por las 
cuotas obrero patronales y el presupuesto federal; el segundo a partir de recursos fiscales y cuotas familiares; y el tercero financiado por seguros privados o pago directo. Desde luego, esta estructura contempla la inserción del sector privado en los tres subsistemas, fundamentalmente a partir de dos mecanismos: el SPS y la subrogación de servicios.

Se puede observar una configuración diferente del Sistema Nacional de Salud, con una clara separación entre instituciones administradoras del financiamiento y prestadoras de servicios, que a diferencia del esquema anterior, en el que sólo podían ser públicas, en la actualidad también pueden ser privadas. Esto plantea para la población una segmentación del sistema que, aunque sigue teniendo como característica fundamental la separación entre población asegurada y no asegurada, abre para ambas, una diversidad de servicios a los que estos grupos podrán acceder a partir de seguros privados, de acuerdo a su capacidad de pago.

Esta nueva estructura del SNS, cuyos cambios radican en la inserción del sector privado en sus diferentes niveles y la emergencia definitiva en México del denominado mix público-privado de la atención médica, se concreta en el SPS, el cual tiene como rasgo intrínseco el traslado de recursos del sector público al privado (9). La nueva estructura del SNS anticipa una recomposición de la segmentación de la población con base en una nueva forma de acceso diferencial a los servicios de salud. Así, el acceso de la población a la atención médica ha sido redefinido, conformándose un grupo bajo la cobertura de la seguridad social, compuesto fundamentalmente por trabajadores de la economía formal de media y baja cotización (los afiliados al IMSS y al ISSSTE) ; otro grupo, proveniente de la economía informal, población pobre no asegurada que, en el mejor de los casos, tendrá acceso a los servicios brindados por las instituciones estatales de salud, compitiendo por la atención con los grupos bajo el SPS; y un tercer grupo, que a partir del SPS, accederá a servicios privados de acuerdo a su capacidad de pago, compuesto por los grupos de mayor cotización a la seguridad social, sectores de la economía informal y la población de clase media no asegurada.

La parquedad de los recursos a la que se ha sometido desde hace más de veinte años al sub-sector público, ha producido un deterioro de los servicios. Dentro del nuevo esquema, este sub-sector queda en desventaja para competir con el privado. Así, el análisis de la evolución de los recursos y de los servicios durante la década pasada confirma la conformación de un sector público mucho menos fuerte que en el pasado, sobre todo en lo relacionado con la cobertura 
de servicios de segundo y tercer nivel, en correspondencia con la expansión del sub-sector privado a expensas del crecimiento de diversas formas de aseguramiento (10).

Antecedentes y evolución del SPS

El SPS inició su fase piloto en octubre de 2001 en cinco estados de la República (Aguascalientes, Campeche, Colima, Jalisco y Tabasco), y constituyó el "brazo operativo" del Sistema de Protección Social en Salud a partir del $1^{\circ}$ de enero de 2004, cuando entraron en vigor las adiciones a la Ley General de Salud(11), que sustentan las acciones de protección social en salud que ofrecerán las entidades federativas. Según la versión oficial, su principal objetivo es sustituir el pago de bolsillo de las familias por el prepago, condición indispensable para el financiamiento de los seguros médicos y para la expansión del sector privado.

La población objetivo fue las familias de los seis primeros deciles de la distribución de ingreso que no son derechohabientes de la seguridad social, residentes en el territorio nacional y que tuvieran acceso a unidades de salud con capacidad y calidad suficiente para ofrecer la prestación de los servicios médicos (12).

Los servicios ofrecidos por el SPS se dividen en servicios esenciales y servicios de alto costo. Los primeros se contemplan en el Catálogo de Servicios Esenciales de Salud (CASES) y se ofrecen en unidades de atención ambulatoria y hospitales generales. Los servicios de alto costo se ofrecen en hospitales de especialidad. Esto constituye un cambio notable en la concepción del derecho a la salud, que ahora circunscribe la prestación de servicios a un catálogo de beneficios médicos de primer nivel y a otros de hospitalización para especialidades básicas en el segundo nivel de atención.

El SPS se postula como público y voluntario. Sin embargo, en los hechos este programa más bien es de carácter obligatorio y su costo es cubierto mediante subsidio federal proveniente de recursos fiscales y de cuotas de los beneficiarios por concepto de afiliación según decil de ingreso. La contribución familiar se complementa con una aportación estatal mínima ( 0.5 veces la cuota social) y una aportación federal (1.5 veces la cuota social) procedente del actual Fondo de Aportaciones para los Servicios de Salud (FASSA, Ramo 33), que ya financia la mayor parte de las actividades de salud de las entidades federativas. 
Los servicios esenciales se financian con recursos que se concentran en el Fondo de Aportaciones para los Servicios de Salud a la Persona (FASSP), mientras que los servicios de alto costo lo hacen a través de un Fondo de Protección contra Gastos Catastróficos (FPGC). Este fondo cubre un paquete de servicios definidos por medio de un análisis de costo-efectividad que, supuestamente, será puesto al día cada año con base en los cambios del perfil epidemiológico, el desarrollo tecnológico y la disponibilidad de recursos.

Además del FPGC, el SPS cuenta con otros dos fondos: el primero de ellos, equivalente al $2 \%$ de la suma de la cuota social, la contribución solidaria federal y la contribución solidaria estatal que debería de estar destinado a la promoción de la infraestructura en las comunidades pobres; el segundo, igual al 1\% de esa misma suma, orientado a cubrir las fluctuaciones en la demanda y los pagos retrasados dentro de los estados. Con respecto a las cuotas de los afiliados, se determinó fuesen menores a los gastos de bolsillo reportados por la Encuesta de Ingreso Gasto de los Hogares por decil de ingreso (13). En la Tabla 1 se muestran los conceptos de cuotas de acuerdo al decil de ingreso.

Aparentemente, esta estructura de cotización conforma un sistema solidario pues la cuota es progresiva según el nivel de ingreso de cada familia y es complementada por el subsidio federal para cubrir el costo de la prima. Ante este panorama cabe preguntar ¿de dónde se financiarán los diversos fondos de la propuesta? Es lógico pensar que serán subsidiados con recursos federales o estatales, de tal manera que esta propuesta devela una intención de segmentar y deteriorar más las arcas financieras de la seguridad social.

\section{Cobertura}

Al cierre del año 2004, un total de 30 entidades suscribieron el acuerdo. Durante el primer bimestre del 2005 se incorporaron Durango y el Distrito Federal. Cabe mencionar que en esta última entidad, la implementación del seguro fue sin consenso de las autoridades sanitarias locales. Al respecto, la entonces secretaria de salud de la Ciudad de México, Cristina Laurell, fue contundente al afirmar que el Seguro Popular incrementaba la segmentación del sistema de salud pues dividía a la población sin seguridad social en dos nuevos grupos. Además, resultaba ser una propuesta poco viable para los habitantes de esta entidad federativa, dado que desde 2001 se había puesto en marcha el programa de servicios médicos y medicamentos gratuitos (14). 
En lo que respecta a la cobertura del Seguro Popular se pueden encontrar numerosas irregularidades e incongruencias. El índice compuesto de "cobertura efectiva", entendida como "el uso de un servicio, condicionado a una necesidad verdadera y ajustado por la calidad de la intervención”, elaborado y utilizado por Lozano, Gakidou y colaboradores (15) para evaluar el avance del Seguro Popular a nivel nacional, como lo ha demostrado Laurell (16), contiene varias deficiencias. Las conclusiones a las que llegan dichos autores a partir de los datos obtenidos con el mencionado índice de cobertura efectiva no son sorpresivos ni revelan nada nuevo: los estados más pobres tienen menor cobertura efectiva y los que destinan más gasto público a salud tienen mayor cobertura efectiva.

Tabla 1. Cuota por familia según decil de ingreso y porcentaje en relación al ingreso promedio

\begin{tabular}{|c|c|c|c|c|c|c|c|c|c|}
\hline \multirow[b]{2}{*}{ Decil } & \multicolumn{3}{|c|}{ Ingreso comiente trimestral por hogar } & \multirow[b]{2}{*}{$\begin{array}{c}\text { Cuota } \\
\text { trimestral }\end{array}$} & \multirow{2}{*}{$\begin{array}{c}\% \text { en } \\
\text { relación } \\
\text { con } \\
\text { ingreso } \\
\text { promedio } \\
\text { trimestral }\end{array}$} & \multirow[b]{2}{*}{$\begin{array}{c}\text { Cuota } \\
\text { semestral }\end{array}$} & \multirow{2}{*}{$\begin{array}{c}\text { \% en } \\
\text { relación } \\
\text { con } \\
\text { ingreso } \\
\text { promedio } \\
\text { semestral }\end{array}$} & \multirow[b]{2}{*}{$\begin{array}{l}\text { Cuota } \\
\text { anual }\end{array}$} & \multirow{2}{*}{$\begin{array}{c}\text { \% en } \\
\text { relación } \\
\text { con } \\
\text { ingreso } \\
\text { promedio } \\
\text { anual }\end{array}$} \\
\hline & $\begin{array}{l}\text { Promedio } \\
\text { trimestral }\end{array}$ & $\begin{array}{l}\text { Promedio } \\
\text { semestral }\end{array}$ & $\begin{array}{l}\text { Promedio } \\
\text { anual }\end{array}$ & & & & & & \\
\hline 1 & 3537 & 7074 & 14148 & 65 & 1,8 & 130 & 1,8 & 260 & 1,8 \\
\hline ॥I & 6157 & 12314 & 24628 & 95 & 1,5 & 190 & 1,5 & 380 & 1,5 \\
\hline III & 8395 & 16790 & 33580 & 160 & 1,9 & 320 & 1,9 & 640 & 1,9 \\
\hline IV & 10707 & 21.414 & 42828 & 285 & 2.7 & 570 & 2,7 & 1140 & 2,7 \\
\hline V & 13284 & 26568 & 53136 & 475 & 3.6 & 950 & 3,6 & 1900 & 3,6 \\
\hline VI & 16511 & 33022 & 66044 & 600 & 3,6 & 1200 & 3,6 & 2400 & 3,6 \\
\hline VI & 20611 & 41222 & 82444 & 790 & 3,8 & 1580 & 3,8 & 3160 & 3,8 \\
\hline VIII & 26209 & 52418 & 104836 & 945 & 3,6 & 1890 & 3,6 & 3780 & 3,6 \\
\hline IX & 37502 & 75004 & 150008 & 1260 & 3,4 & 2520 & 3,4 & 5040 & 3,4 \\
\hline$x$ & 90224 & 180448 & 360896 & 1575 & 1,7 & 3150 & 1,7 & 6300 & 1,7 \\
\hline
\end{tabular}

Fuente: Adaptado de Cámara de Diputados (CD). 2005. Programa Seguro Popular. México: Centro de Estudios Sociales y de Opinión Pública, p.43.

Sin embargo, una dificultad importante al querer evaluar el desempeño del SP es que los datos estadísticos proporcionados por la propia Secretaría de Salud (SS) son incongruentes. El número de hogares sujetos de afiliación (los que no cuentan con seguridad social) que utiliza la Comisión Nacional de Protección Social en Salud (CNPSS), dependiente de la Secretaría de Salud, para medir el grado de avance del SP, no corresponde con el total de población no asegurada por entidad federativa que la misma Secretaría de Salud reconoce a través de su Dirección General de Información en Salud (17). Por ejemplo, según los datos de la Dirección General de Afiliación y Operación, perteneciente a la CNPSS, en los estados de Tabasco y Colima habría ya en estos momentos una tasa de hogares afiliados al SP de 128 \% y $129 \%$, respectivamente. Sin embargo, estos mismos datos no reconocen la existencia de 42185 personas en el estado de Tabasco y 9102 personas en el estado de Colima no aseguradas que sí son reconocidas por la Dirección General de Información en Salud. Lo 
más sorprendente es que ambas Direcciones, pertenecientes a la SS, reconocen como fuente de sus estimaciones al XII Censo de Población y Vivienda, elaborado por el Instituto Nacional de Estadística, Geografía e Informática en el año 2000. Y así en cada entidad federativa: cientos de miles de personas sin seguridad social no son contempladas por la CNPSS como sujetos del derecho a la protección a la salud.

Si esto es así en un estado pequeño como Colima, y en un estado con el mayor gasto público en salud en la historia, como Tabasco (ambos pioneros del SPS), ¿qué podríamos esperar en estados de extrema pobreza y marginación como Chiapas, Oaxaca, Guerrero y Michoacán? De los 3632 291 chiapanecos, en su mayoría indígenas, sin seguridad social, sólo han afiliado a menos de la tercera parte (32,9\%); de los 2827006 oaxaqueños sin seguro social, sólo han afiliado al 24,8 \%; de los 2471050 guerrerences sin seguridad social, sólo han afiliado al 26,3\%; y de los 2997567 michoacanos sin seguridad social, sólo han afiliado al 12,3 \%. Más allá de discursos entusiastas sobre la “democratización de la salud” y de “evidencias” tecnocráticas, la realidad de la salud en México está muy lejos de encaminarse al $100 \%$ de población cubierta para el 2010, como los defensores del SPS vaticinan.

Algo parecido ha demostrado la Auditoría Superior de la Federación (ASF) del Congreso mexicano al encontrar que "se incumplirá el compromiso de alcanzar el $100 \%$ de cobertura en el año 2010, toda vez que, con la incorporación del 14,3 \% anual, se alcanzará el 94,7\% (11 986 227) de las 12649905 familias que la SS ha previsto para ese año (2006); además, para entonces la ASF estimó que la población objetivo ascenderá a 14 039620 , lo que significa que 2053393 no tendrán acceso a las prestaciones y servicios del sistema"(18).

Tendencias del Seguro Popular de salud

Durante el año 2006 fueron publicados en The Lancet una serie de artículos en los que prácticamente se proclama el éxito rotundo del SPS (19,20); sin embargo, existen algunos hechos que cuestionan el prematuro "éxito" y la viabilidad del SPS como aspirante a convertirse en el tercer régimen de seguridad social del país.

El SPS deberá cubrir en el año 2010 al 50 \% de la población mexicana con un crecimiento promedio anual del 14,3\%; sin embargo, dado que en la primera fase no se construyó infraestructura alguna, la atención prestada fue prácticamente a expensas de los servicios de atención de la red pública de la SS y de los sistemas estatales de salud, ya de por sí insuficiente. En lo financiero, 
se prevé una gran vulnerabilidad de los presupuestos estatales, pues el presupuesto federal fluirá hacia los estados en función de la cantidad de familias aseguradas y por lo tanto, el que registre menos afiliación, será el que reciba menor presupuesto. De esta forma, en Chiapas, uno de los estados con menor infraestructura, el 80\% de la población se ubica en los deciles I y II que no pagan prima; en contraste, en Nuevo León, este tipo de habitantes representan el 29\% y el resto está adscrito a algún esquema de seguridad social; esto plantea para el sistema una inequidad estructural de origen.

Por otro lado, comparando las cuotas de la Seguridad Social con las del SPS, se observa que las cuotas de la primera van de 7 a 100 dólares, mientras que las del segundo se encuentran tasadas entre 60 y casi 1000 dólares (16). Además, otra desventaja es que en la SS, la exención de cuotas obreras se hace con ingresos menores a tres salarios mínimos lo que corresponde al V decil del SPS; considerando que en este último seguro la exención de pago sólo se realiza en los dos primeros deciles, el gasto resulta mucho más oneroso para las familias.

Los recursos materiales y humanos también son un problema pues, hasta la fecha, se han construido 1792 unidades ambulatorias y unos cuantos hospitales con solamente 12 a 20 camas que, por cierto, no se ubican en áreas críticas. Atendiendo a la estructura del gasto, no se visualiza la solución a este problema, pues el $15 \%$ está destinado a contratación de personal, el 59 \% a medicamentos y solamente el $26 \%$ es destinado a inversión de capital (16).

Los paquetes de servicios también representan limitaciones serias pues, además de que las acciones son elegidas bajo el criterio de costo/efectividad, no contemplan varios problemas de salud que ocupan las primeras causas de morbilidad y mortalidad como infarto, cáncer y accidentes severos. Inicialmente sólo fueron contemplados 78 padecimientos, pero la lista tuvo que ser revisada en dos ocasiones bajo criterios poco claros; actualmente cubre 225 intervenciones (21) y en muchos casos, ítems que aparecen como nuevos, representan solamente una especificación de varias modalidades de un solo padecimiento.

Otra gran limitación se deriva de que, en contra de la lógica de la evolución de la enfermedad, el paquete contempla algunos casos que implican ciertos grados de gravedad. Es decir, si los pacientes empeoran se ven en grave riesgo de no ser atendidos. Dentro de estos rótulos se encuentran padecimientos como enfermedad isquémica del corazón y traumatismo craneoencefálico. En cuanto al FPGC, de las 17 causas elegidas, se han atendido solo 4 tipos de padecimientos, dando como resultado que existan fondos que no son utilizados. 
Finalmente, la evaluación en la que se basó la proclama del éxito del SPS a partir del método basado "en evidencias" ha sido severamente cuestionada por Laurell (16) debido a las siguientes cuestiones:

- Solo 5 de los 34 autores a los que se hace referencia son ajenos al grupo de trabajo del entonces secretario de salud;

- Fueron aplicados índices compuestos y no se sabe el peso específico de cada aspecto considerado;

- Se ocultan datos y fuentes originales, lo que da lugar a que varias estimacio nes se realicen sobre datos inexistentes o no verificables;

- La evaluación de la "cobertura efectiva” se basa solamente en 18 interven ciones, de las cuales 10 no contemplan la calidad de la intervención y 9 son autoreportadas;

- Las categorías y procedimientos estadísticos no son explicados;

- Los cálculos pueden estar sesgados debido a que se trata de poblaciones que ya contaban con infraestructura sanitaria antes de la entrada en operación del SPS.

Además, el SPS tiene un costo que están pagando todos los mexicanos con recursos fiscales y se prevé que progresivamente los gobiernos estatales y la población vayan asumiendo la totalidad de dicho gasto en un periodo de cinco años (22). Es decir, las entidades que no puedan cubrir el fideicomiso correspondiente tendrán que recurrir a las instituciones de servicios privados para compensar la falta de infraestructura lo que probablemente repercutirá en incremento en las cuotas de los afiliados.

El SPS no atiende las causas que empobrecen a las familias mexicanas como la hospitalización, las intervenciones quirúrgicas y el acceso a medicamentos para tratar enfermedades como el cáncer cérvico-uterino. Además, tampoco incluye “enfermedades persistentes" en un plazo máximo de 18 meses, es decir, si alguien tiene un padecimiento de varios años atrás o que fue diagnosticado médicamente hace tiempo, tampoco las cubre, y hasta el cuadro de medicamentos esta limitado.

Consideraciones finales

Después de 20 años de la aplicación de las políticas económicas de austeridad en México, los resultados muestran que las prioridades de desarrollo social que dice tener el gobierno van en sentido contrario. Como observamos, la política de la salud se ha encaminado a la creación de un mercado para el sector privado, a través de dos estrategias: a) hacer que los servicios públicos sean 
percibidos como insuficientes y de mala calidad (lo cual incrementa la demanda de los servicios privados); y b) contar con formas de financiamiento para el sector privado.

Aunado a esto, la reducción del gasto en salud ha causado que el sector dedique cada vez más recursos al pago de la nómina y menos al gasto de inversión, hecho que ha influido de manera negativa en la ampliación de la infraestructura y en el equipamiento de las unidades.

Por otra parte, frente a una grave problemática de la situación sanitaria en México, que se debate entre la fuerte reducción presupuestal, el monopolio de hospitales privados y el encarecimiento desmedido de los medicamentos, el gobierno neoliberal implementa el Seguro Popular de Salud (SPS), programa que adolece de una propuesta médico-clínica de bienestar social cuyo propósito real es implantar el modelo llamado de participación público-privada.

El SPS, pieza clave en la reestructuración del Sistema Nacional de Salud, muestra dos problemas fundamentales: 1) no es viable, pues hasta ahora se trata primordialmente de una propuesta que no cuenta con la infraestructura necesaria para brindar servicios de calidad a la población "asegurada”; y 2) la cobertura de los riesgos es muy limitada y condicionada.

Debido a que no hay una perspectiva médica en las reformas estructurales, el SPS representa un programa discrecional, presidencial y focalizado que resta fondos a las instituciones grandes de la seguridad social. El SPS profundiza la descentralización de los servicios de salud con el ingrediente de que ahora las entidades federativas van a tener que aportar una cantidad igual o superior a la que aporte la federación (23), obligándolas, en muchos casos, a realizar adecuaciones presupuestarias en detrimento de la calidad de la atención.

Esta situación constituye para el futuro inmediato una segmentación del SNS que determinará nuevas condiciones diferenciales del acceso de la población a los servicios médicos. Estas condiciones aumentarán la inequidad en salud y contribuirán a incrementar la gran desigualdad social prevaleciente en México 


\section{REFERENCIAS}

1. Laurell AC. No hay pierde: Todos pierden. La reforma a la ley del IMSS. México, D.F.: Instituto de la Revolución Democrática; 1996.

2. Instituto Mexicano del Seguro Social (IMSS). Ley del Instituto Mexicano del Seguro Social. México, D.F.: IMSS; 1997.

3. Programa de Reforma del Sector Salud 1995-2000; [Internet] Disponible en: http:// info4.juridicas.unam.mx/ijure/nrm/1/336/default.htm?s=iste Consultado Noviembre 2007.

4. Secretaria de Salud (SS). Programa Nacional de Salud 2001-2006. México D.F.: SS; 2001.

5. Giugale M, Lafourcade O, Nguyen V, comps. Mexico: A Comprehensive DevelopmentAgenda for the New Era. Washington D.C.: World Bank; 2001.

6. Fundación Mexicana para la Salud (FUNSALUD). La reforma de la salud en México. México, D.F.: FUNSALUD; 2001.

7. World Health Organization (WHO). World Health Report 2000, Health Systems: Improving Performance. Estatistic annex. Geneva: WHO; [Internet]. Disponible en formato PDF en: <http://www.who.int/ whr2001/2001/archives/2000/en/ contents.htm> Consultado Octubre de 2007.

8. Soberón G. La participación del sector privado. En: La reforma de la salud en México. México, D.F.: FUNSALUD; 2001. p. 43-55.

9. Tamez S, Moreno P. La seguridad social en América Latina. En: Enrique de la Garza comp. Tratado Latinoamericano de Sociología del Trabajo. México D.F.: El Colegio de México, Universidad Autónoma Metropolitana, FLACSO, Fondo de Cultura Económica; 2000. p. 515-534.

10. Tamez S, Valle RI. Desigualdad social y reforma neoliberal en salud. Revista Mexicana de Sociología, 2005; 67(2): 321-356.

11. Congreso de la Unión. Ley General de Salud. Diario Oficial de la Federación; 7 de febrero de 1984.

12. Secretaría de Salud. Programa Nacional de Salud; [Internet] Disponible en: http:// evaluacion.salud.gob.mx/publicaciones/pns_2001-2006/pns2001-006.pdf Consultado Noviembre 2007.

13. Cámara de Diputados. Programa Seguro Popular. México: Centro de Estudios Sociales y de Opinión Pública; 2005. p. 43

14. Gómez-Dantés O, Ortiz M. Seguro Popular de Salud: Siete perspectivas. Salud Pública de México 2004; 46(6): 586.

15. Gakidou E, Lozano R, González-Pier E, Abbott-Klafter J, Barofsky JT, Bryson-Cahn C, et al. Evaluación del impacto de la reforma mexicana de salud 2001-2006: un informe inicial. Salud Pública de México, 2007; 49(suplemento 1): 88-109.

16. Laurell AC. Health System Reform in Mexico: A Critical Review. International Journal of Health Services, 2007; 37(3): 515-535.

17. Secretaria de Salud. Dirección General de Información en Salud; [Internet] Disponible en: http:// dgis.salud.gob.mx/ Consultado Noviembre 2007.

18. Auditoría Superior de la Federación. Informe del Resultado de la Revisión y Fiscalización Superior de la Cuenta Pública 2005, Informe Ejecutivo; [Internet] Disponible en: http://nww.asf.gob.mx/ trans/Informes/IR2005i/Indice/Auditorias.htm Consultado Noviembre 2007.

19. Frenk J. Brindging the divide: Global lessons from evidence-based health policy in Mexico. Lancet, 2006; 368: 954-961.

20. Frenk J, González-Pier E, Gómez-Dantés O, Lezama MA, Knaul FM. Comprehensive reform to improve health system performance in Mexico. Lancet, 2006; 368:1524-1534.

21. Secretaría de Salud. Seguro Popular de Salud. Beneficios; [Internet] Disponible en: http:/l www.seguro-popular.salud.gob.mx/contenidos/menu_beneficios/ beneficios_inicio.html Consultado Noviembre 2007.

22. Tetelboin C, Granados A. Lectura del Programa Nacional de Salud 2001-2006 en México. Argumentos, 2003; 45: 2-23.

23. Leal G. El Seguro Popular en el D.F; [Internet] Disponible en: http://www.imagenmedica.com.mx/ datos/modules.php?name=News\&file=article \&sid=477 Consultado Noviembre 2007. 